\title{
Evolução mensal da cota fluviométrica do Rio Xingu em Altamira-PA associada aos eventos El Niño e La Niña
}

\author{
Monthly evolution of streamlevel of the Xingu River in Altamira-PA associated with \\ the El Niño and La Niña events
}

\author{
Vânia dos Santos Franco ${ }^{1}$, Everaldo B. De Souza ${ }^{2}$ Amanda N. Pinheiro', \\ Thaiane S. da Silva Dias ${ }^{1}$, Franklin T. M. de Azevedo ${ }^{1}$, Jaqueline C. do Carmo Santos ${ }^{1}$ \\ ${ }^{1}$ Programa de Pós-Graduação em Ciências Ambientais, Universidade Federal do Pará (UFPA), Belém-PA \\ ${ }^{2}$ Instituto Tecnológico Vale (ITV), Belém-PA
}

\begin{abstract}
Resumo
Apresenta-se, neste trabalho, uma contribuição à hidroclimatologia da Amazônia, em particular do regime fluviométrico mensal na sub-bacia do rio Xingu em Altamira-PA (Amazônia oriental), em associação aos episódios ENOS registrados nas últimas três décadas (1979-2012). Em termos climatológicos, a fluviometria do rio Xingu apresenta regime de cheia nos meses de março a maio (com pico máximo de $682 \mathrm{~cm}$ em abril) e regime de vazante nos meses de agosto a outubro (com pico mínimo de $260 \mathrm{~cm}$ em setembro). Os resultados das composições para anos de atuação de El Niño (La Niña) evidenciaram a manifestação de padrões de convecção tropical anomalamente enfraquecidos (intensificados) nas regiões de atuação da ZCIT e da ZCAS, que resultaram, consequentemente, na ocorrência de anomalias negativas (positivas) de precipitação em grande parte da Amazônia, explicando, assim, o regime hidrológico predominantemente abaixo (acima) da média no rio Xingu em Altamira-PA.
\end{abstract}

Palavras-chave: Hidroclimatologia da Amazônia, El Niño/La Niña, precipitação, ZCAS, ZCIT.

\begin{abstract}
This work presents a contribution to the Amazon hydroclimatology, in particular the monthly streamlevel variability of the Xingu river sub-basin in Altamira-PA (eastern Amazon) in association with ENSO episodes recorded in the last three decades (1979-2012). In climatological terms, the highest level regime of the Xingu river occurs during March to May (with a maximum peak of $682 \mathrm{~cm}$ in April) and the lowest level regime happens from August to October (with minimum peak of $260 \mathrm{~cm}$ in September). The results of the composites for years of occurrence of El Niño (La Niña) evidenced patterns of tropical convection anomalously weakened (intensified) within ITCZ and SACZ regions which consequently resulted in the occurrence of anomalies negative (positive) precipitation in most of the Amazon, thus explaining the hydrological regime predominantly below (above) average on the Xingu river in Altamira-PA.
\end{abstract}

Keywords: Amazon hydroclimatology, El Niño/La Niña, precipitation, SAZC, ITCZ 


\section{Introdução}

A precipitação dentro da região amazônica é uma variável que apresenta alta variabilidade no tempo e espaço, associada à influência de diferentes sistemas de mesoescala, escala sinótica e de grande escala (ROCHA, 2001).

De acordo com DE SOUZA et al (2000), a variabilidade pluviométrica sazonal da Amazônia apresenta dois períodos distintos, conhecidos como período chuvoso e seco ou menos chuvoso, sendo influenciados pelo fenômeno acoplado oceano-atmosfera sobre o Pacífico tropical, o ENOS - El Niño-Oscilação Sul, o qual desempenha papel importante na variabilidade climática Amazônica (KOUSKY et al, 1984; MARENGO 1992; LIEBMANN e MARENGO, 2001), ora aumentando os processos de ocorrência da precipitação, ora inibindo o desenvolvimento da convecção profunda e, com isso, diminuindo a precipitação (FISCH et al, 1998). O El Niño/La Niña são as fases positiva/negativa do fenômeno ENOS e representam o aquecimento/resfriamento anômalo da Temperatura da Superfície do Mar (TSM) na região do Pacífico tropical (DE SOUZA et al, 2000).

FU et al (2001) apresentaram resultados observacionais sobre a influência do Pacífico nas precipitações da Amazônia oriental e constataram que, durante os eventos La Niña, as precipitações se configuram com padrões regionais acima da média climatológica durante o período chuvoso, enquanto que, durante os eventos El Niño, os padrões de chuva são essencialmente inversos.

DE SOUZA et al. (2005) consideraram dois cenários climáticos contrastantes, denominados favorável (La Niña no Pacífico e anomalias de TSM negativa/positiva no Atlântico norte/sul) e desfavorável (El Niño no Pacífico e TSM anomalamente positiva/negativa no Atlântico norte/sul) ao regime pluviométrico da estação chuvosa na Amazônia. O cenário favorável (desfavorável) relaciona-se com a predominância de circulação atmosférica ascendente (descendente) nas regiões da célula de Walker/Hadley equatorial, que intensificam (inibem) a convecção tropical da Zona de Convergência Intertropical (ZCIT) e da Zona de Convergência do Atlântico Sul (ZCAS), resultando, consequentemente, num padrão de precipitação significativamente abundante (deficitário) na Amazônia/Nordeste Brasileiro.

A distribuição espacial da precipitação influencia diretamente o regime hidrológico da bacia amazônica e o comportamento de subida (cheia) e descida (vazante) dos níveis ou cota dos rios amazônicos (SANTOS, 2008). Existe uma grande necessidade da realização de estudos sobre a relação dos fenômenos expostos anteriormente com o comportamento dos regimes de cheia/vazante dos rios da Amazônia, particularmente na resposta da hidrologia regional (na escala de sub-bacia). Desse modo, o presente trabalho tem o objetivo de analisar a evolução mensal da cota fluviométrica do Rio Xingu (com foco em Altamira-PA), fazendo uma associação com os anos de atuação dos fenômenos El Niño e La Niña. Além disso, investigam-se os padrões de convecção associada à manifestação da ZCIT e da ZCAS, que induzem a ocorrência de precipitação sobre a sub-bacia do Xingu. Essa sub-bacia hidrográfica da Amazônia oriental destaca-se pelo alto potencial hidroelétrico devido ao seu grande volume de água e relevo acidentado, propiciando condições ideais para geração de energia.

\section{Material e métodos}

A área de estudo do presente trabalho é ilustrada na Figura 1, que engloba a sub-bacia do rio Xingu sobre a Amazônia oriental. Esta sub-bacia possui uma área de $531.250 \mathrm{~km}^{2}$, sendo que $216.823 \mathrm{~km}^{2}(40,8 \%)$ são sobre o estado do Mato Grosso e os restantes, $59,2 \%$, sobre o estado do Pará, com uma área de, aproximadamente, $314.427,790 \mathrm{~km}^{2}$. No Pará a sub-bacia do Xingu corresponde a $25,1 \%$ da área do estado e abrange os municípios de Altamira, São Félix do Xingu, Senador José Porfírio, Vitória do Xingu, Brasil Novo, parte de Medicilândia, Placas e Anapú. Os principais cursos d'água formadores dessa bacia são os rios Xingu, Irirí, Curuá, Fresco, Bacajá, Caeté, Ximxim e Chiche (MELO, 2004).

As análises do regime hidrológico são baseadas nos dados de cota ou nível fluviométrico médio mensal registrado nas réguas limnimétricas instaladas no rio Xingu, no município de Altamira-PA. Estes dados foram obtidos na Agencia Nacional de Águas (ANA) e dispõe-se de uma série temporal de dados no período de 1979 a 2012.

Usam-se dados mensais de precipitação numa grade recortada sobre a Amazônia, com resolução horizontal de $0,5^{\circ}$ (aproximadamente $55 \mathrm{~km}$ ), no período de Janeiro de 1979 a Dezembro de 2012. Estes dados foram gerados através do sistema de análise de dados observacionais do Climate Prediction Center (CPC) da NOAA-USA e é baseado, inteiramente, em dados de estações meteorológicas proveniente da rede nacional e estadual (SILVA et al 2007). Visando analisar os padrões de convecção tropical, utilizam-se dados mensais de radiação de onda longa emergente - ROL (LIEBMANN e SMITH, 1996), numa grade com resolução horizontal de 2,5\%, disponível no período de 1979 a 2012.

Com a finalidade de se investigar a contribuição da ocorrência de precipitação regional para o regime hidrológico de cheia/vazante do rio Xingu, foram geradas as séries temporais da precipitação espacialmente mediada na região-chave da sub-bacia do Xingu, situada à montante da estação de Altamira-PA, conforme ilustra a Figura 1 (quadrado lilás). Da mesma forma, foram geradas as médias espaciais de ROL, representativas da região de atuação da ZCIT, sobre o Atlântico equatorial adjacente à costa amazônica (Figura 1, quadrado azul), e da ZCAS (Figura 1, quadrado verde), sobre o centrosul da Amazônia, visando à investigação dos padrões 


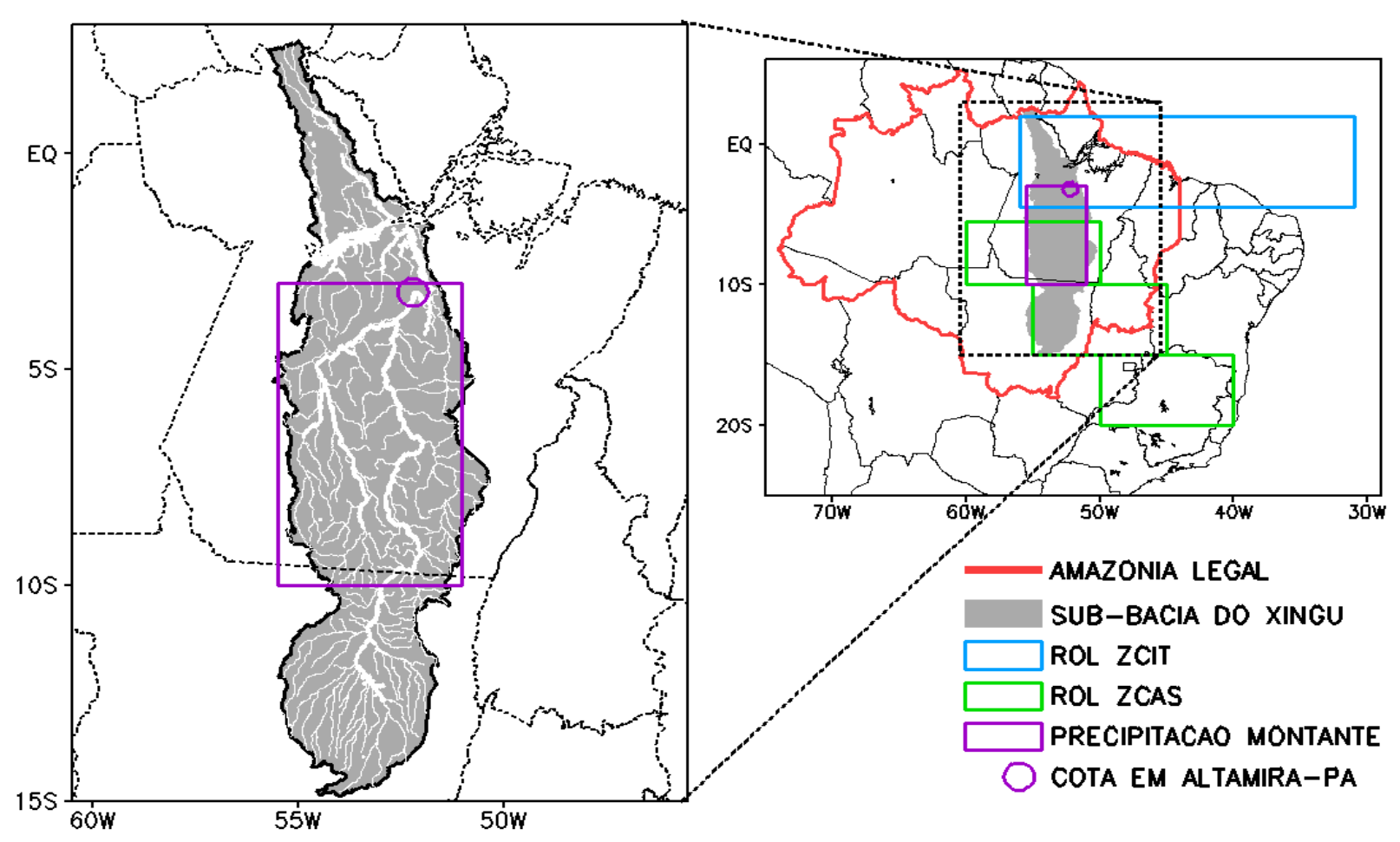

Figura 1 - Mapa à esquerda: localização da área de estudo destacando a sub-bacia do rio Xingu (área cinza) com as isolinhas em branco indicando a rede de drenagem hidrológica e o círculo lilás mostrando a estação fluviométrica de Altamira-PA. Mapa à direita: Domínio sobre o Brasil tropical destacando a Amazônia Legal e as regiões onde foram obtidas as médias espaciais de precipitação na sub-bacia do Xingu e de ROL na regiã̃o de atuação da ZCIT e da ZCAS

de convecção que induzem a ocorrência de chuva na sub-bacia do Xingu.

As médias climatológicas mensais, referentes ao período 1979/2012, da cota fluviométrica em Altamira foram calculadas e, assim, foi definido o ano hidrológico (ciclo anual completo do regime hidrológico de cheia e vazante) do rio Xingu, o qual se processa de novembro de um ano até outubro do ano posterior.

Os anos de ocorrência das fases do ENOS baseiam-se nos critérios de SOUZA et al (2005) e, também, do Grupo de Clima do Centro de Previsão do Tempo e Estudos Climáticos (CPTEC/INPE). Os anos de El Niño (ano +1) analisados foram 1983, 1987, 1992, 1995, 1998, 2007 e 2010 e os anos de La Niña foram 1985, 1989, 1996, 1999, 2000, 2008 e 2011.

O procedimento de análise do comportamento da fluviometria consiste em investigar a evolução mensal da cota do rio Xingu, em Altamira, considerando a composição de todos os eventos (média representativa dos 7 eventos El Niño e 7 eventos La Niña). A anomalia ou diferença entre as composições de El Niño e La Niña e a média climatológica foi calculada para cada mês do ano hidrológico, com a finalidade de se verificar qual mês apresenta maior variação mensal entre os eventos, os quais, geralmente, associam-se com padrões anômalos de cheias ou vazantes. Também foram calculadas as anomalias de ROL nas regiões da ZCIT, da ZCAS e da precipitação na sub-bacia do Xingu, à montante de Altamira para as composições de El Nino e La Niña, com a finalidade de complementar as análises do regime fluviométrico na sub-bacia.

\section{Resultados}

A Figura 2 mostra a evolução da cota mensal correspondente à média climatológica do período 1979-2012 (MED-CLIM, linha preta) e composições dos anos de El Niño (MED-EN, linha vermelha) e La Niña (MED-LN, linha azul). Analisando o comportamento da MED-CLIM, observa-se que o regime de subida do rio Xingu em Altamira se inicia em novembro, com cota de $298 \mathrm{~cm}$ e demora em torno de quatro meses até atingir o pico da cheia sazonal no trimestre de março a maio (sendo que o nível máximo ocorre em abril com $682 \mathrm{~cm}$ ). A partir de abril, o nível do rio começa a descer progressivamente, até atingir o pico da vazante sazonal no trimestre de agosto a outubro (o nível mais baixo ocorre em setembro, com $260 \mathrm{~cm}$ ).

A comparação entre as composições representativas de todos os episódios El Niño (MED-EN) e La Niña (MED-LN), em relação à MED-CLIM, também é 
ilustrada na Figura 2. Nota-se um comportamento essencialmente inverso no regime hidrológico do rio Xingu em Altamira entre os anos de El Niño e La Niña com níveis fluviométricos, caracterizando-se, respectivamente, abaixo e acima da média climatológica durante a maior parte do ano hidrológico. Visualmente percebe-se que a composição de El Niño exibe valores abaixo do normal, notadamente, entre os meses de janeiro e setembro. $\mathrm{Na}$ composição de La Niña, os valores ficam levemente acima da cota climatológica, sendo que se verifica a antecipação do pico máximo da cheia sazonal de abril para março, prosseguindo com o comportamento acima da média até agosto.

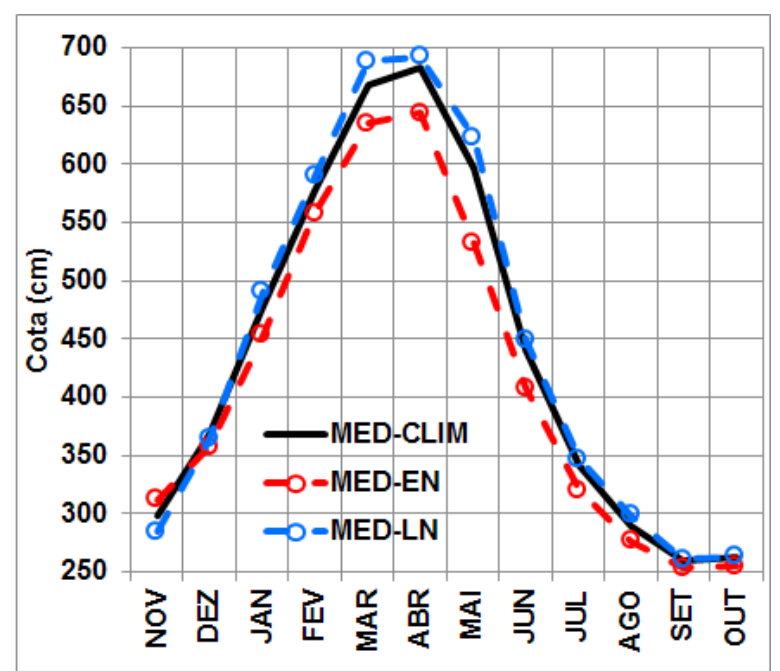

Figura 2 - Ciclo anual das cotas do rio Xingu em Altamira-PA mostrando a evolução mensal do nível fluviométrico $(\mathrm{cm})$ correspondente à média climatológica do período 1979-2012 (MED-CLIM, linha preta) e composições dos anos de El Niño (MED-EN, linha vermelha) e La Niña (MED-LN, linha azul)

A Figura 3 mostra os resultados das composições El Niño e La Niña em termos da anomalia ou desvio absoluto (em cm) e, também, em termos do desvio percentual (em \%), ambos relativos à MED-CLIM. Em geral, o impacto do ENOS no regime hidrológico do rio Xingu é preponderantemente maior para os eventos El Niño, quando comparado aos eventos La Niña. Na composição El Niño, as cotas anomalamente negativas variam de 10 a $24 \mathrm{~cm}$ nos meses de dezembro a fevereiro, se intensificam para 33 a $39 \mathrm{~cm}$ em março e abril e atingem o pico negativo de $64 \mathrm{~cm}$ abaixo do normal em maio, em seguida diminuindo para $33 \mathrm{~cm}$ em junho, 21 $\mathrm{cm}$ em julho e $12 \mathrm{~cm}$ em agosto. Tais valores correspondem a percentuais de -3 a $-6 \%$ relativos a climatologia nos meses de dezembro a abril, com pico de $-11 \%$ em maio e gradativa diminuição para -7 e $-6 \%$ em junho e julho. Por outro lado, a cota acima da média durante os anos de La Niña indicam valores percentuais entre
$+1,5$ e $+3 \%$ nos meses de janeiro a agosto, sendo que o pico de $+4,5 \%$ ocorre em maio.

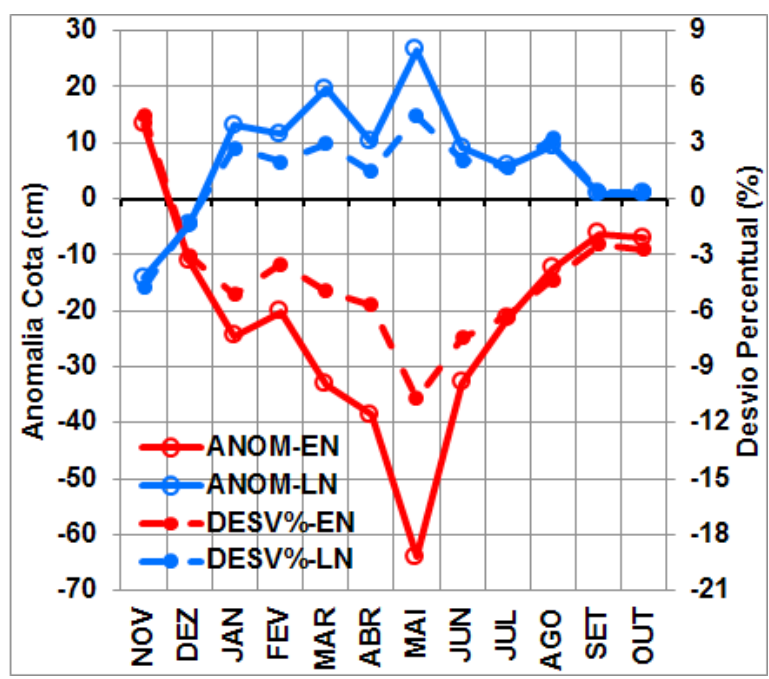

Figura 3 - Evolução mensal da anomalia ou desvio absoluto (linhas contínuas, em cm) e desvios percentuais (linhas tracejadas, em \%) da cota do rio Xingu correspondente às composições de El Niño e La Niña

Baseado nos resultados da Figura 4, apresenta-se a caracterização mensal dos padrões de convecção tropical da ZCIT e da ZCAS, bem como da contribuição da ocorrência de precipitação na sub-bacia hidrográfica à montante do ponto de Altamira-PA, associada às condições de regime hidrológico abaixo/acima do normal durante os anos de El Niño/La Niña.

Em geral, os anos de El Niño relacionam-se com a inibição da atividade convectiva sobre o Brasil tropical. Em termos da banda convectiva da ZCIT sobre o Atlântico equatorial sul, adjacente à costa Amazônica, verifica-se, na Figura 4, a presença de anomalias positivas de ROL (indicando convecção abaixo da média), em torno de 4 $\mathrm{W} / \mathrm{m}^{2}$ em dezembro, se intensificando para valores de $8 \mathrm{~W} / \mathrm{m}^{2}$ em janeiro e fevereiro, diminuindo para, aproximadamente, $6 \mathrm{~W} / \mathrm{m}^{2}$ em março e abril e apresentando pico de $12 \mathrm{~W} / \mathrm{m}^{2} \mathrm{em}$ maio, regredindo, novamente, para $5 \mathrm{~W} / \mathrm{m}^{2}$ em junho e $2 \mathrm{~W} / \mathrm{m}^{2}$ em julho. Similarmente, os padrões anômalos de convecção da ZCAS sobre a porção centro-sul da Amazônia, mostrados na Figura 4, indicam valores predominantemente positivos ao longo dos meses do ano hidrológico. Observam-se anomalias de ROL em torno de $8,4 \mathrm{~W} / \mathrm{m}^{2}$ em novembro, em torno de 3 e $4 \mathrm{~W} /$ $\mathrm{m}^{2}$ em dezembro, janeiro e fevereiro, e entre 6 e $7 \mathrm{~W} / \mathrm{m}^{2}$ de março até junho. Tais configurações de ROL positivo são consistentes com a evolução de anomalias negativas de precipitação na região à montante de Altamira, com valores de $-10 \mathrm{~mm}$ em novembro, intensificando para $-13 \mathrm{~mm}$ em dezembro e fevereiro, com pico de $-19 \mathrm{~mm}$ em janeiro, gradativa diminuição para $-10 \mathrm{~mm}$ em março e para -4 e $-6 \mathrm{~mm}$ entre abril e maio. Esses resultados explicam a manifestação de regime hidrológico abaixo 
da média no rio Xingu, na região de Altamira, durante a manifestação de episódios El Niño.

Por outro lado, no que concerne aos padrões anômalos convecção tropical durante os anos de La Niña, evidencia-se um comportamento convectivo intensificado (ROL negativo) da ZCIT e da ZCAS (exceto em fevereiro) na maior parte dos meses do ano hidrológico. Na região da ZCIT, os valores de ROL oscilam em torno de -4 em novembro, dezembro e ferreiro, em, aproximadamente, -8 durante janeiro, março e abril, com pico de -13 em maio e gradativa retração para -6 em junho e julho. Similarmente, evidenciam-se valores de ROL negativo na região da ZCAS, entre -2 e -4 de dezembro a maio (exceto em fevereiro que apresenta valor positivo de 0,3 ). Estas condições de atividade convectiva anomalamente intensificada (ROL negativo) associam-se à ocorrência de anomalias positivas de precipitação regional, com valores evoluindo de $4 \mathrm{~mm}$ em novembro, $2 \mathrm{~mm}$ em dezembro, $9 \mathrm{~mm}$ em janeiro, retração para o valor neutro de $-0,1$ $\mathrm{mm}$ em fevereiro, com intensificação para 8 e $9 \mathrm{~mm}$ nos meses de março, abril e maio. Essas evidências suportam as condições de regime hidrológico acima da média, verificado na sub-bacia do Xingu, particularmente, nas regiões de Altamira-PA, durante os anos de La Niña.

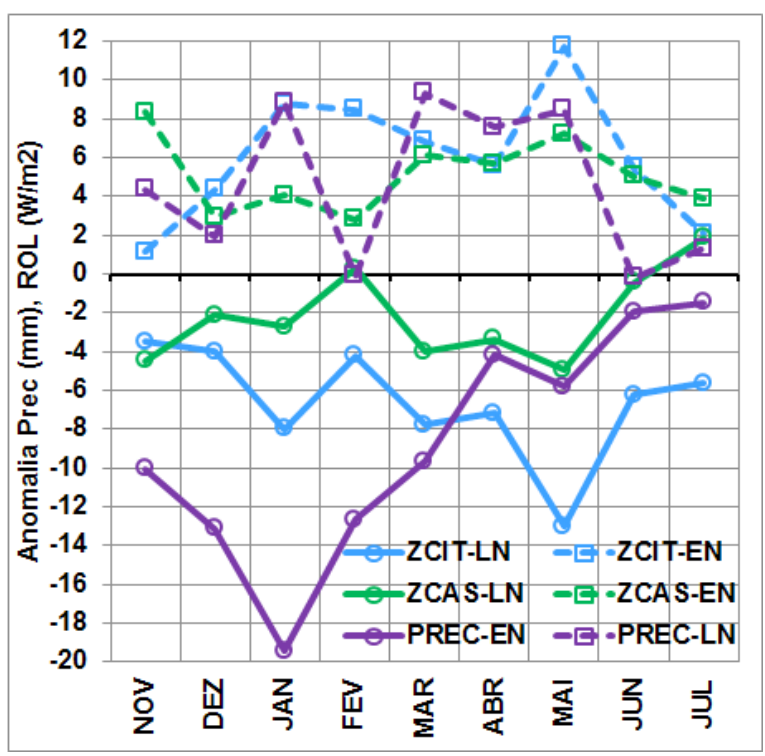

Figura 4 - Evolução mensal das anomalias de precipitação $(\mathrm{mm})$ na sub-bacia do Xingu e de $\mathrm{ROL}\left(\mathrm{W} / \mathrm{m}^{2}\right)$ nas regiões da ZCIT e ZCAS correspondentes as composições de El Niño (linhas tracejadas) e La Niña (linhas sólidas)

\section{Conclusão}

Diversos estudos anteriores abordaram os impactos dos episódios ENOS sobre a variabilidade climática da Amazônia, reportando condições de estação chuvosa abaixo da média em anos de El Niño e acima da média em anos de La Niña (e.g., MARENGO, 1992; DE SOUZA et al, 2000, 2005, entre outros), entretanto tais análises foram enfatizadas numa escala espacial, englobando grandes áreas e numa escala de tempo sazonal. O presente trabalho apresenta uma contribuição à hidroclimatologia da Amazônia, com enfoque na escala regional, particularmente, na sub-bacia do Xingu e nas variações da fluviometria, pluviometria e convecção tropical na escala de tempo mensal, sendo que os resultados obtidos são consistentes com aqueles trabalhos anteriores.

Os resultados observacionais do presente trabalho permitiram evidenciar as seguintes conclusões:

Em termos climatológicos, a fluviometria do rio Xingu, na região de Altamira-PA, apresenta o regime de cheia nos meses de março a maio (com pico máximo de 682 $\mathrm{cm}$ em abril) e regime de vazante nos meses de agosto a outubro (com pico mínimo de $260 \mathrm{~cm}$ em setembro), portanto, com uma amplitude anual entre a cota mínima e máxima em torno de $422 \mathrm{~cm}$;

Considerando a composições dos episódios ENOS registrados nas últimas três décadas (1979 a 2012), evidenciou-se, durante os anos de La Niña, a ocorrência de regime hidrológico abundante no rio Xingu, com cota fluviométrica em torno de 2 a $4 \%$ acima da média, prolongando-se nos meses de janeiro a agosto Em termos quantitativos, os impactos dos eventos El Niño são mais intensos (comparados aos da La Niña), com a preponderância de regime hidrológico deficitário ao longo do ano hidrológico, tal que o nível do rio Xingu oscila entre -3 e $-7 \%$ abaixo da média nos meses de dezembro a agosto, sendo que o pico negativo de $-11 \%$ se processa em maio;

Os anos de El Niño (La Niña) se associam com a manifestação de padrões de convecção tropical anomalamente enfraquecidos (intensificados) nas regiões de atuação da ZCIT e da ZCAS, que resultaram, consequentemente, na ocorrência de anomalias negativas (positivas) de precipitação em grande parte da Amazônia, explicando, assim, o regime hidrológico predominantemente abaixo (acima) da média no rio Xingu, em Altamira-PA.

\section{Referências}

DE SOUZA, E.; KAYANO, M.; TOTA, J.; PEZZI, L.; FISCH, G.; NOBRE, C. On the influences of the El Niño, La Niña and Atlantic dipole pattern on the Amazonian rainfall during 1960-1998. Acta Amazônica, v. 30, n. 2, p. 305-318, 2000.

DE SOUZA, E.B.; KAYANO, M.T.; AMBRIZZI, T. Intraseasonal and submonthly variability over the eastern Amazon and Northeast Brazil during the autumn rainy season. Theoretical and Applied Climatology, v. 81, p. 177-191, 2005.

FISCH, G.F.; MARENGO, J.A.; NOBRE, C.A. Uma revisão geral do clima da Amazônia. Acta Amazônica, v. 28, n. 2, p. 101-126, 1998. 
FU, R.; DICKINSON, R.E.; CHEN, M., WANG, H. How do tropical sea surface temperatures influence the seasonal distribution of precipitation in the equatorial Amazon? Journal of Climate, v. 14, p. 4003-4026, 2001.

KOUSKY, V.E., KAYANO, M.T., CAVALCANTI, I.F.A. A review of the Southern Oscillation: oceanic, atmospheric circulation changes and related anomalies. Tellus $\mathrm{v}$. 36A, p. 490-504, 1984.

LIEBMANN, B., MARENGO. J. A. Interannual variability of the rainy season and rainfall in the Brazilian Amazonia. Journal of Climate 14: 4308-4318. 2001.

LIEBMANN, B.; SMITH, C.A. Description of a complete (interpolated) outgoing longwave radiation dataset. Bulletin of the American Meteorological Society, v. 77, p. 1275-1277, 1996.

MARENGO JA. Interannual variability of surface climate in the Amazon basin. International Journal of Climatology 12: 853-863. 1992.

MELO, A.P., Aplicação de modelos estatísticos para previsão de níveis no rio Xingu em Altamira. Trabalho de Conclusão de Curso - Universidade Federal do Pará - UFPA, 2004.

ROCHA, E. J. P. Balanço de Umidade e Influências de Condições de Contorno Superficiais Sobre a Precipitação da Amazônia. 2001. 210 f. (Tese de Doutorado) - Instituto Nacional de Pesquisas Espaciais, INPE, São Paulo, 2001.

SANTOS, D. M. Análise e Modelagem Hidrometeorológica na Bacia do Tocantins em Marabá-PA. 118f. Dissertação de Mestrado. Universidade Federal do Pará. Belém Pará. 2008.

SILVA, V. B. S., V. E. KOUSKY, W. SHI, R. W. HIGGINS. An improved gridded historical daily precipitation analysis for Brazil. Journal of Hydrometeorology, v. 8, p. 847-861, 2007. 